\title{
BMJ Open Analysis of English general practice level data linking medication levels, service activity and demography to levels of glycaemic control being achieved in type 2 diabetes to improve clinical practice and patient outcomes
}

Adrian Heald, ${ }^{\oplus 1,2}$ Mark Davies, ${ }^{3}$ Mike Stedman, ${ }^{3}$ Mark Livingston, ${ }^{4}$ Mark Lunt, ${ }^{2}$ Anthony Fryer, ${ }^{5}$ Roger Gadsby ${ }^{6}$

To cite: Heald A, Davies M, Stedman M, et al. Analysis of English general practice level data linking medication levels, service activity and demography to levels of glycaemic control being achieved in type 2 diabetes to improve clinical practice and patient outcomes. BMJ Open 2019;9:e028278. doi:10.1136/ bmjopen-2018-028278

- Prepublication history and additional material for this paper are available online. To view these files, please visit the journal online (http://dx.doi. org/10.1136/bmjopen-2018028278).

Received 20 December 2018 Revised 18 June 2019 Accepted 19 June 2019

Check for updates

(C) Author(s) (or their employer(s)) 2019. Re-use permitted under CC BY-NC. No commercial re-use. See rights and permissions. Published by BMJ.

For numbered affiliations see end of article.

Correspondence to

Dr Mark Davies;

mdavies@resconsortium.com

\section{ABSTRACT}

Objective Evaluate relative clinical effectiveness of treatment options for type 2 diabetes mellitus (T2DM) using a statistical model of real-world evidence within UK general practitioner practices (GPP), to quantify the opportunities for diabetes care performance improvement. Method From the National Diabetes Audit in 2015-2016 and 2016-2017, GPP target glycaemic control (TGC$\% \mathrm{HbA} 1 \mathrm{c} \leq 58 \mathrm{mmol} / \mathrm{mol}$ ) and higher glycaemic risk ( $\mathrm{HGR}-\% \mathrm{HbA} 1 \mathrm{c}$ results $>86 \mathrm{mmol} / \mathrm{mol}$ ) outcomes were linked using multivariate linear regression to prescribing, demographics and practice service indicators. This was carried out both cross-sectionally (XS) (within year) and longitudinally (Lo) (across years) on 35 indicators. Standardised $\beta$ coefficients were used to show relative level of impact of each factor. Improvement opportunity was calculated as impact on TGC \& HGR numbers. Results Values from 6525 GPP with 2.7 million T2DM individuals were included. The cross-sectional model accounted for up to $28 \%$ TGC variance and $35 \%$ HGR variance, and the longitudinal model accounted for up to $9 \%$ TGC and $17 \%$ HGR variance. Practice service indicators including $\%$ achieving routine checks/blood pressure/cholesterol control targets were positively correlated, while demographic indicators including $\%$ younger age/social deprivation/white ethnicity were negatively correlated. The $\beta$ values for selected molecules are shown as (increased TGC; decreased HGR), canagliflozin (XS 0.07;0.145/Lo 0.04;0.07), metformin (XS 0.12;0.04/Lo -;-), sitagliptin (XS 0.06;0.02/Lo 0.10;0.06), empagliflozin (XS-;0.07/Lo 0.09;0.07), dapagliflozin (XS $-; 0.04 / L 0-; 0.4)$, sulphonylurea (XS -0.18;-0.12/Lo-;-) and insulin (XS-0.14;0.02/ Lo-0.09;-). Moving all GPP prescribing and interventions to the equivalent of the top performing decile of GPP could result in total patients in TGC increasing from 1.90 million to 2.14 million, and total HGR falling from 191000 to 123000 .

Conclusions GPP using more legacy therapies such as sulphonylurea/insulin demonstrate poorer outcomes, while those applying holistic patient management/use of newer molecules demonstrate improved glycaemic outcomes. If

\section{Strengths and limitations of this study}

- This study provides insights into the inter-relationship between the portfolio of type 2 diabetes mellitus (T2DM) medicines and service interventions in primary care and patient outcomes in real-world settings not addressed by randomised control trial approaches.

- The databases used in this study, including the National Diabetes Audit and primary care prescribing database, offer a comprehensive summary of participating practice activities in the care of T2DM within primary care.

- Multivariate regression analysis is an established methodology in identifying associations between a selected range of variables and outcomes in actual clinical practice.

- One limitation is that association cannot be assumed to being causal, with potential confounding factors such as comorbidities and other local behavioural factors being contributors to the results.

- Restricted use of certain factors such as new medications being introduced may have limited the ability of this model to identify their significance.

all GPP moved service levels/prescribing to those of the top decile, both TGC/HGR could be substantially improved.

\section{INTRODUCTION}

The National Diabetes Audit (NDA) of England ${ }^{1}$ reported that over the period 2006/2007 to $2016 / 2017$, the diagnosed population of people with type 2 diabetes mellitus (T2DM) in England rose by $22 \%$ from 2.3 million to 2.8 million and the proportion of people with T2DM achieving target glycaemic control (TGC) with glycosylated haemoglobin (HbAlc) $\leq 7.5 \%(58 \mathrm{mmol} / \mathrm{mol})$ fell from 
$66.5 \%$ to $65.7 \%$. In the same period, those patients at higher glycaemic risk (HGR) with HbA1c $>10.0 \%$ (86 $\mathrm{mmol} / \mathrm{mol}$ ) decreased from $7.0 \%$ to $6.7 \%$.

A recent report, Prescribing for Diabetes England 20062007 to $2016 / 2017,{ }^{2}$ describes a $70 \%$ increase in total expenditure on drugs used to treat T2DM over that period from $£ 160$ to $£ 272$ million/year respectively, with the main cost drivers being newer agents such as glucagon-like peptide-1 (GLP-1) agonists, dipeptidyl peptidase-4 (DPP-4) inhibitors and sodium-glucose cotransporter-2 (SGLT-2) inhibitors.

With growing pressures on local primary care services, optimising how general practitioner practices (GPP) deliver care to T2DM patients is critical. For optimisation of care to take place, and resources to be targeted at the right interventions, a best practice benchmark is required defining the factors more likely to provide improved outcomes. We recently reported that some classes of diabetes therapies such as SGLT-2 inhibitors and GLP-1 analogues are associated with a larger proportion of individuals achieving $\mathrm{HbAlc}<58 \mathrm{mmol} / \mathrm{mol}^{3}{ }^{3}$ That paper reported that in year-on-year analysis, SGLT-2 inhibitor prescribing was associated with a greater reduction in patients at HGR than occurred with GLP-1 analogues. Targeting the more effective agents is therefore fundamental to longer term affordability.

This subsequent paper seeks to determine at GPP level the differences in the relation of individualmedicines and service interventions to outcomes, by determining factor correlation with the proportion of T2DM patients who are well controlled (\%HbA1c Last Result $\leq 58 \mathrm{mmol} / \mathrm{mol}$ ), and those who have poor glycaemic control $(\% \mathrm{HbAlc}$ Last Result $>86 \mathrm{mmol} / \mathrm{mol}$ ). The objective is to identify a 'performance profile' of factors positively correlated with T2DM patient outcomes which can provide a benchmark against which GPP can compare and improve their management of, and prescribing for, T2DM. This profile of factors can also support a more informed debate around the prioritisation criteria for investment in existing and future T2DM therapies and interventions that can deliver greater relative benefits.

\section{METHODS}

This latest analysis is based on current publicly available diabetes NHS data sets including the NDA, ${ }^{1}$ Quality and Outcome Framework (QoF) ${ }^{4}$ and National Primary Care Prescribing database ${ }^{5}$ together with Public Health Observatory data. ${ }^{6}$ A consolidated data set was created and extracted using MS Excel 64 bit Power Pivot and extracted data were analysed using the Analyse-it add in - the same multivariate regression methodology as previously published papers. This analysis linked medicines and other therapeutic interventions at English GPP level with glycaemic control outcomes as defined by relative proportions of patients falling within the given HbAlc control categories as reported in the NDA.
Multivariate regression analysis is a statistical procedure for analysis of data involving more than one type of measurement or observation. The approach uses simultaneous analysis of variables to determine the level of relation between factors, using p-values and standardised $\beta$-values, the latter referring to a standardised regression coefficient that compares the strength of the effect of each individual independent variable to the dependent variable. This method can identify real-world correlations of one variable with another variable-in this case T2DM interventions with disease control. Longitudinal studies may give more precise estimates of temporal changes or treatment effects than cross-sectional studies of the same size. When risk factors vary more across space at a fixed moment in time than at a fixed location across time, cross-sectional studies will tend to give more precise estimates of risk factor effects. Hence, both cross-sectional and longitudinal approaches were used.

A range of medicine and non-medicine intervention factors were identified related to type 1 and type 2 diabetes from NDA and QoF data sets. The medicine classes and individual molecules identified included:

- Metformin.

- Sulphonylurea.

- Pioglitazone.

- Insulins.

- SGLT-2 inhibitors (dapagliflozin, empagliflozin, canagliflozin).

- DPP-4 inhibitors (alogliptin, linagliptin, saxagliptin, sitagliptin, vildagliptin).

- GLP-1 agonists (dulaglutide, exenatide day+weekly), liraglutide, lixisenatide).

For medicines, all data were adjusted for 'per patient' using 'defined daily dose' (or 'DDD', which is the average daily dose of a particular medicine as defined by the WHO). The approach allowed overall patients presribed to be calculated, but could not show individual patient prescribing profiles either in combination (except where the British National Formulary (BNF) code itself referred to a combined therapy) or the actual doses being applied. The $\%$ of people with type 2 on insulin was estimated by subtracting the amount of insulin for type 1 (estimated by multiplying the recorded number of type 1 in the practice register by 50 units/day and 365) from the total and then dividing the remainder by 100 units/day and 365 . This figure was then taken as \% of the type 2 recorded register. Other non-medicine service factors were also included in the analysis to determine the level to which these would predict outcomes. These included NDA metrics such as blood pressure, cholesterol levels, renal function (creatinine and urine albumin/creatinine ratio), foot checks, body mass index and smoking. Non-HbAlc outcomes were also included in the analysis including \% patients with blood pressure $\leq 140 / 80$ and $\%$ patients with total serum cholesterol $<4 \mathrm{mmol} / \mathrm{L}$.

Impact on outcomes was defined by two measures:

- \% of patients within GPPs at TGC (HbA1c $\leq 58 \mathrm{mmol} /$ mol) and 
- \% of patients within GPPs at HGR (HbAlc $>86 \mathrm{mmol} /$ $\mathrm{mol})$.

While 35 different factors (see online supplementary appendix) were originally tested, only 20 of those achieved correlation p-values below 0.05 , and were subsequently included in the results. In relation to drug combinations, metformin plus other oral agents are included in the analysis although these preparations are little used in comparison with individual prescribed molecules. ${ }^{8}$ Therefore, their contribution to the overall regression analysis is likely to be minimal. In relation to double (eg, metformin+gliclazide), triple (eg, metformin+SGLT2+GLP-1 analogue) and quadruple therapy, the contributions of each agent are accounted for separately in the analysis.

As a real-world analysis, resultant correlations could be influenced by confounding factors. Including potential confounders in the regression may decrease the bias of the treatment effect; however, adding more variables can decrease statistical power in small samples because it increases the variance (spread) around the regression estimate by decreasing the degrees of freedom (df). ${ }^{9}$ The model was designed to reduce the potential effect of these by conducting analysis on consolidated populations of over 100 people within each GPP practice. In addition, the wide range of variables of different classes taken from different sources over different time periods meant potential unidentified confounders were more likely to be accounted for in one or more of the identified measured variables, reducing likely confounding in the regression analysis. Applying both longitudinal and cross-sectional models to evaluate the same data sets is also more likely to reduce unaccounted influencing factors. However, despite approaches to reduce these confounders, the possibility of unidentified influences on correlation results remains.

Another challenge with diabetes is the long-term nature of the disease. Outcomes status can be influenced by a number of historical factors such as choice of medicine and quality of interventions such as reviews. This means, for example, that patients currently prescribed insulin could be due to previous poor choice of oral medicines at an earlier point in the patient's disease cycle, rather than an intrinsic aspect of their disease. Therefore, two approaches were applied in the regression analysis:

- Cross-sectional: This explored the association of patient outcomes with different levels of prescribing and other factors across different GPPs for the latest year of data where regression analysis was applied to the cohort of GPP in 2016-2017. This mode of analysis is more sensitive to differences in prescribing between GPP.

- Longitudinal: This explored the association of patient outcomes with different levels of prescribing and other factors within the same GPP across 2 years of data, regression analysis being applied between the net change in outcome levels and the change in factor level between 2015-2016 and 2016-2017. This mode of analysis is more sensitive to newer agents for which there is significant change in year-on-year prescribing or agents whose use is declining year-on-year.

Two different metrics were calculated for both TGC and HGR in both cross-sectional and longitudinal views.

- Standardised beta coefficient: For all indicators with $\mathrm{p}$ value $<0.05$, the standardised beta coefficient was calculated to show the direction and level of association of change in that indicator against the chosen outcome. The sign was adjusted so that a positive value was associated with positive change in that outcome that is, increase in TGC and reduction in HGR.

- Potential outcome numbers affected by a change in indicator value: As GPP demographics cannot be changed, they were not included in the analysis. As all indicators can be presented as a ratio to the total T2DM population, applying the calculated regression coefficient to a difference between indicator values and multiplying that by the total national T2DM population would give an estimate of the change in numbers of patients moving into TGC or out of HGR if GPP were to achieve the same pattern of clinical behaviours as those GPP in the top performance decile for patient outcomes. This assumes that the observed positive or negative association had some causal impact, accepting that confounders cannot be ruled out in a study of this nature.

For the cross-sectional analysis, the regression coefficient was applied to the difference between the median population value and the 10th and 90th percentile values (as indicated by the sign) of all the GPP being evaluated for that indicator, providing a best practice correlation standard.

For the longitudinal analysis, the regression coefficient was applied to the difference in indicator value to the previous year, multiplied against the total T2DM population to provide an indication of the association of the annual change of that indicator with change in outcomes.

As the indicators are associated with a total number of patients, the number of patients required to achieve a unit change in the outcome from that indicator can be calculated. This figure, the number needed to treat (NNT), can be obtained through an inversion of the regression coefficient for that indicator.

\section{Patient involvement}

No patients, carers or lay people were involved in the design or conduct of this study, which only used publicly available data.

\section{RESULTS}

\section{Cross-sectional analysis}

Cross-sectional data from 2016 to 2017 operational year were analysed which included 6614 GPP (table 1) who had participated in the NDA, each with more than 100 T2DM patients per practice. These results were based on a total registered population for England of 57.9 million. The 
Table 1 Participation in the study

\begin{tabular}{lllll}
\hline GPP participation in 2016-2017 & QoF & NDA & $\begin{array}{l}\text { NDA >100T2DM } \\
\text { patients }\end{array}$ & $\begin{array}{l}\text { GPP also participating in 2015- } \\
\text { 2016 }\end{array}$ \\
\hline Number of practices & 7366 & 6873 & 6614 & 5488 \\
Population & 57925672 & 54953783 & 53837296 & 45588943 \\
DM register & 3112478 & 2958290 & 2927864 & 2471551 \\
NDA T2 & & 2699945 & 2683420 & 2267835 \\
NDA T2 HbA1c $\% \leq 58$ & & $66.9 \%$ & $66.9 \%$ & $66.8 \%$ \\
NDA T2 HbA1c $\%>86$ & $6.7 \%$ & $6.7 \%$ & $6.7 \%$
\end{tabular}

GPP, general practitioner practice; NDA, National Diabetes Audit; QoF, Quality and Outcome Framework.

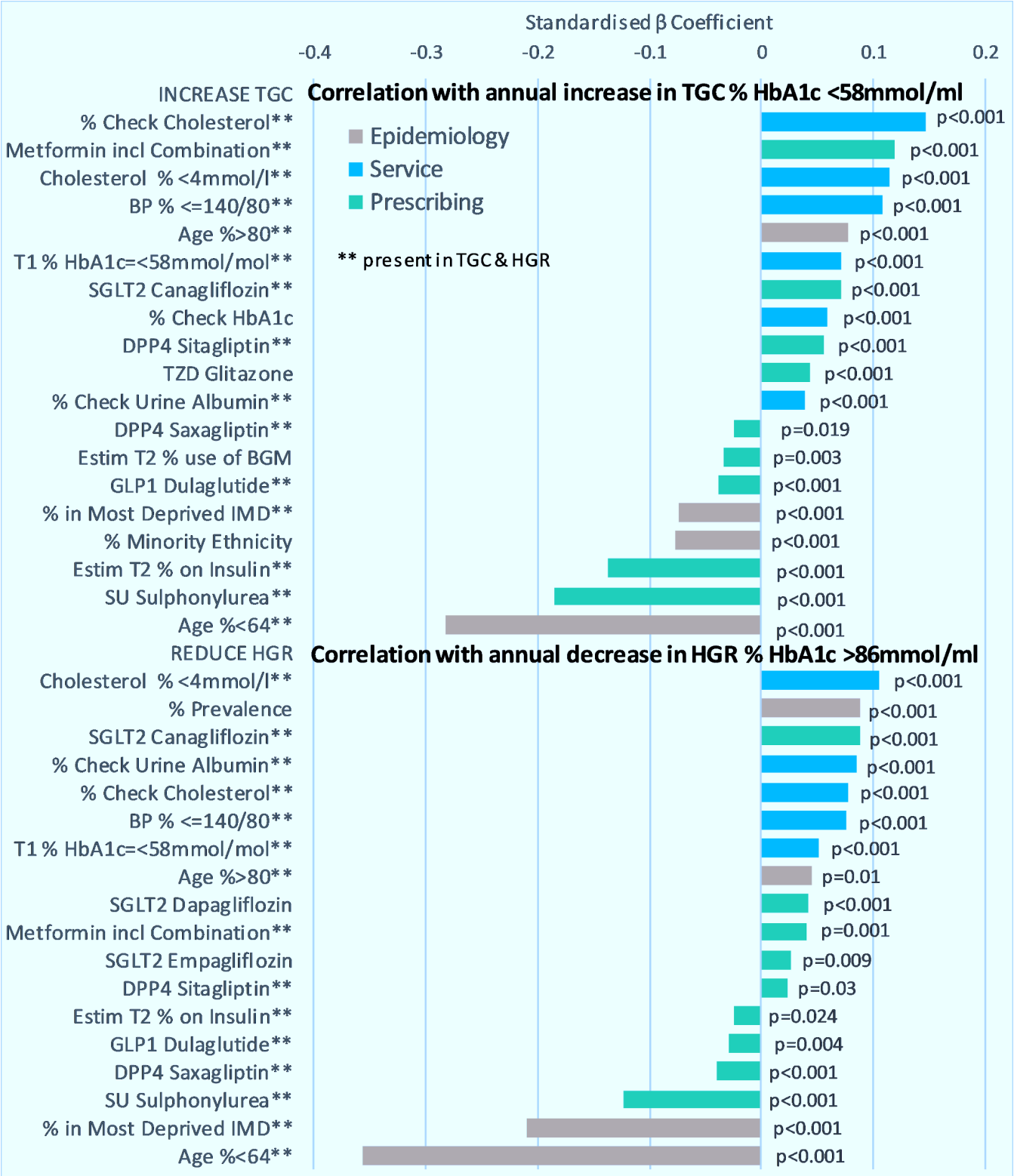

Figure 1 Cross-sectional-standardised beta value ( $\beta$ ). BP, blood pressure; DPP-4, dipeptidyl peptidase-4 inhibitor; GLP-1, glucagon-like peptide-1 agonist; HGR, higher glycaemic risk; SGLT-2, sodium-glucose cotransporter-2 inhibitor; TGC, target glycaemic control. 
multiple regression model results (figure 1) accounted for $29 \%$ of the variance in TGC and $35 \%$ of the variance in HGR groups.

Use of specific medication showing positive association to both TGC control (ie, increasing numbers in this group) and HGR (decreasing numbers in this group) included metformin (single and combination prescribing) (TGC $\beta=0.119 ; p<0.001 ;$ HGR $\beta=0.04 ; p<0.001$ ), canagliflozin (TGC $\beta=0.07 ; p<0.001 ;$ HGR $\beta=0.09 ; p<0.001$ ) and sitagliptin (TGC $\beta=0.06 ; p<0.001 ;$ HGR $\beta=0.02 ; p<0.03$ ). Medicines only showing positive correlation with HGR reduced numbers included dapagliflozin (HGR $\beta=0.04$; $\mathrm{p}<0.001)$ and empagliflozin $($ HGR $\beta=0.03 ; \mathrm{p}<0.01)$. Use of sulphonylurea (TGC $\beta=-0.185 ; p<0.001$; HGR $\beta=-0.123$; $\mathrm{p}<0.001$ ) and insulin (TGC $\beta=-0.14 ; \mathrm{p}<0.001$; HGR $\beta=-0.02 ; p<0.001)$ was negatively associated, correlating with decreased numbers at TGC and increased numbers of HGR.

Demographic factors also demonstrated negative impact on both outcomes, including GPP with higher $\%$ of younger age patients (TGC $\beta=-0.28 ; \mathrm{p}<0.001$; HGR $\beta=-0.36 ; p<0.001$ ) and greater social deprivation (TGC $\beta=-0.07 ; p<0.001 ;$ HGR $\beta=-0.21 ; p<0.001)$.

Non-medicine local service activities that showed positive association to both outcome groups included $\%$ completion of cholesterol checks (TGC $\beta=0.15$; $\mathrm{p}<0.001 ;$ HGR $\beta=0.08 ; \mathrm{p}<0.001), \%$ measured cholesterol levels $<4 \mathrm{mmol} / \mathrm{mol}$ (TGC $\beta=0.12 ; \mathrm{p}<0.001 ; \mathrm{HGR} \beta=-0.10$; $\mathrm{p}<0.001)$ and $\%$ blood pressure results $\leq 140 / 80 \mathrm{~mm} \mathrm{Hg}$ (TGC $\beta=0.11 ; p<0.001 ;$ HGR $\beta=0.08 ; p<0.001$ ).

\section{Longitudinal analysis}

Longitudinal data compared results with the previous 2015-2016year. This covered 5488 GPP who had participated in NDA for both years each with more than $100 \mathrm{~T} 2 \mathrm{M}$ patients and included results in 2016-2017 from 2.26 million T2 patients from a total population of 45.6 million. The model (figure 2) identified 9\% of the variance in TGC change and $17 \%$ in HGR change.

Medicines showing positive associations with TGC and HGR included sitagliptin (TGC $\beta=0.1 ; \mathrm{p}<0.001$; HGR $\mathrm{B}=0.07 ; \mathrm{p}<0.001$ ), empagliflozin (TGC $\beta=0.09 ; \mathrm{p}<0.001$; HGR $\beta=0.07 ; p<0.001$ ) and canagliflozin (TGC $\beta=0.04$; $\mathrm{p}<0.001 ;$ HGR $\beta=0.06 ; \mathrm{p}<0.001)$.

Local service activities showing positive association to improved outcomes included \% identification of T2DM on the QoF register (TGC $\beta=0.13$; $p<0.001$; HGR $\beta=0.07$; $\mathrm{p}<0.001$ ), \% completion of foot checks (TGC $\beta=0.12$; $\mathrm{p}<0.001$; HGR $\beta=0.37 ; \mathrm{p}<0.001), \%$ completion of blood pressure checks (TGC $\beta=0.8 ; \mathrm{p}<0.001$ ) and \% HbA1c glycaemic control being achieved in their type $1 \mathrm{DM}$ population (TGC $\beta=0.13 ; \mathrm{p}<0.001 ;$ HGR $\beta=0.07 ; \mathrm{p}<0.001$ ).

\section{Impact of applying cross-sectional findings to population}

The potential impact of GPP across England adjusting prescribing and service practices to be comparable to those of GPP in the top decile of T2DM outcomes performance (figure 3) could bring an estimated 238100 more patients into TGC, equivalent to $15 \%$ improvement. Better delivery of local service factors by ensuring cholesterol and blood pressure checks are comparable to the top 10 percentile level could bring a further 70000 people into TGC. For prescribing, reducing levels of sulphonylurea to 0.33 and use of insulin to $0.073 \mathrm{DDD} /$ T2DM population would increase TGC by 55000 , while increasing metformin to 0.625 and canagliflozin to 0.024 , sitagliptin 0.138 , glitazone to $0.068 \mathrm{DDD} / \mathrm{T} 2 \mathrm{DM}$ population would increase TGC by a further 44000 patients.

Opportunity for improvement can be expressed as NNT for each significant factor, through increases in canagliflozin (3), metformin (11), sitagliptin (13) and decreased sulphonylurea (20). Assuming limited influence of confounding factors, aligning clinical practice with the top decile of GPP identified in this analysis could contribute to almost 70,000 people with T2DM moving out of the HGR category. Of this total, increasing compliance with local service activities such as cholesterol and blood pressure control could contribute to 30,000 moving out of the HGR category; reducing prescribing of sulphonylurea and insulin could contribute to a further 13,000 reduction; while increasing dapagliflozin, canagliflozin, empagliflozin, metformin and sitagliptin could contribute to a further 24,500 reduction in HGR numbers. Opportunity for improvement for HGR is shown as NNT through increases in canagliflozin (6), metformin (11), empagliflozin (14), dapagliflozin (20) and sitagliptin (71); and decrease in sulphonylurea (20).

\section{Applying longitudinal findings to explain actual year on changes}

From the longitudinal analysis (figure 4), there was an equivalent annual increase of 25,235 patients in the TGC group in the total T2DM population from the previous year, with 13,597 coming from factors not included within the study ('Factors not included in study' refers to other factors that are captured in the regression constant and therefore not explicitly identified in the analysis). Of note, 17,085 came from those practices that had increased prescribing in either empagliflozin, linagliptin, alogliptin or canagliflozin. However, practices that reduced levels of foot-checks, cholesterol control and use of glitazone were associated with an estimated reduction of TGC patients by 12,264 .

Opportunity for improvement for TGC is showed as NNT through increases in empagliflozin (4), canagliflozin (5) linagliptin (7) and alogliptin (10). There was also an equivalent increase of 3700 in HGR in the total T2DM population compared with the previous year, while other factors not included in the study reduced numbers by 4540. GPPs who increased prescribing of empagliflozin, canagliflozin, linagliptin, dapagliflozin and alogliptin reduced HGR by a further 7513. For some GPPs however, this improvement in outcomes was offset by failure to perform foot-checks which was associated with an overall HGR increase of 11,455. Foot checks represented one of the most positive and significant correlations with 


\section{$\begin{array}{lllllllllllll}-0.1 & -0.08 & -0.06 & -0.04 & -0.02 & 0 & 0.02 & 0.04 & 0.06 & 0.08 & 0.1 & 0.12 & 0.14\end{array}$}
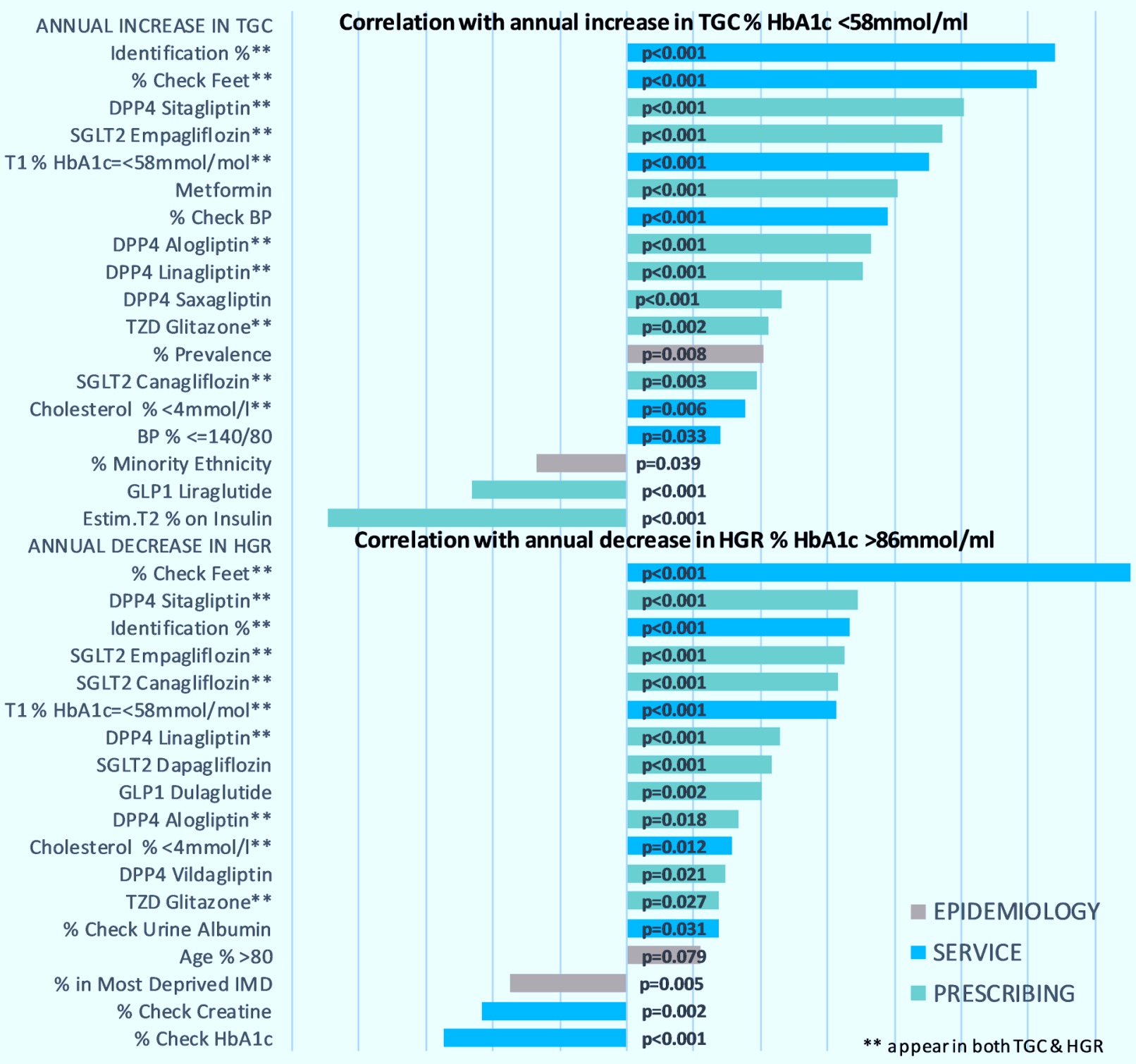

Figure 2 Longitudinal-standardised beta correlation value ( $\beta$ ). BP, blood pressure; DPP-4, dipeptidyl peptidase-4 inhibitor; GLP-1, glucagon-like peptide-1 agonist; HGR, higher glycaemic risk; SGLT-2, sodium-glucose cotransporter-2 inhibitor; TGC, target glycaemic control.

improved diabetes outocmes in GPP. Opportunity for improvement in HGR is shown as NNT through increases in empagliflozin (6), canagliflozin (6), alogliptin (10), linagliptin (12) and dapagliflozin (12).

\section{DISCUSSION}

Optimising the management of diabetes in primary care means optimising the interventions across the primary care diabetes pathway. This multivariate analysis demonstrates that of the measured variables in diabetes management, some medicines and service activities are positively and more significantly associated with improved diabetes outcomes compared with others, both in terms of increasing numbers of patients who are well controlled (TGC group) and reducing numbers of patients who are poorly controlled (HGR group). Broadly speaking, three key factor categories were important in the performance of primary care diabetes care: medicines, service activities and demographics.

Using a novel approach, we report variation between performance in achievement of HbAlc targets between thousands of GPP and separate them by centiles. Our assertions are based around the notion that if even a proportion of GPP were brought up to the performance level of the top $10 \%$ of GPP, then large numbers of people with T2DM could be brought into TGC with 


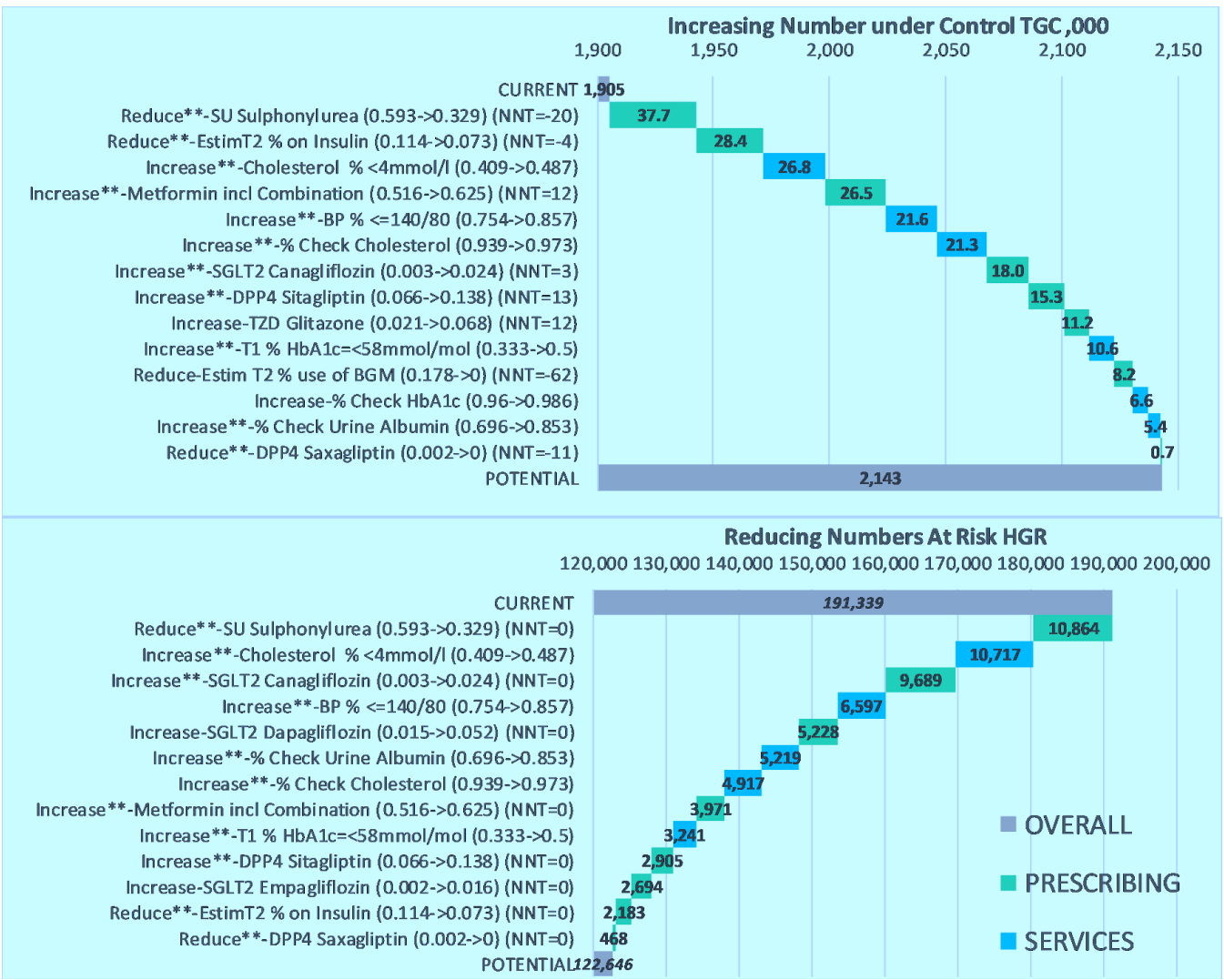

Figure 3 Cross-sectional analysis of potential impact on patient numbers. BP, blood pressure; DPP-4, dipeptidyl peptidase-4 inhibitor; HGR, higher glycaemic risk; SGLT-2, sodium-glucose cotransporter-2 inhibitor; TGC, target glycaemic control.

significant numbers taken out of high glycaemic risk. This is not attempting to attribute direct causation, rather to propose that service organisational change and alteration in prescribing practice are associated with significant improvements in patient outcomes, and likely associated longer term improvement in prognosis and reduction in comorbidities.

Of the medicinal interventions, canagliflozin and metformin showed a particular positive relation in the cross-sectional analysis with better glycaemic outcomes, with the other two SGLT-2 inhibitors empagliflozin and dapagliflozin showing a similar relation in the longitudinal analyses. This supports previous findings of superior glycaemic control with SGLT-2 inhibitor agents as described elsewhere using different methodologies. ${ }^{1011}$

DPP-4 inhibitors were consistently positively correlated across both analysis types, although not to the extent of the SGLT-2 inhibitors. Sulphonylurea and higher use of insulin for T2DM were negatively correlated with patient outcomes. This has implications for the use of sulphonylurea as second line after metformin and supports the position taken by the American Diabetes Association in January 2017 that sulphonylurea should not be second line after metformin. ${ }^{12}$

Of the service activities, in general undertaking NDA checks (including foot checks, blood pressure and cholesterol control) were positively correlated with improved outcomes, while the demographic factors such as deprivation and ethnicity were negatively correlated. Previous studies have indicated that in the UK, population demographics can have a profound impact on health outcomes, including those associated with T2DM. ${ }^{8}$

As a potential guide for GPP wanting to improve their approach to diabetes care, we have shown previously through cross-sectional analysis for the year of NDA data $2016-2017,{ }^{13}$ that moving levels of local service activities and prescribing of metformin and sulphonylurea from the median to the 90th percentile level could be associated with more than 200000 additional patients in TGC and more than 60000 fewer in HGR. The associations between change in SGLT-2 inhibitor and DPP-4 inhibitor prescription at a practice level and the proportions at TGC and HGR may be important, if the link is causative. ${ }^{14}$

In terms of cost, improving glucose control by using newer drugs would increase costs. For example, we speculate that an investment of $£ 50$ million in additional SGLT-2 inhibitors in those practices prescribing around or below the national average to include an additional 100000 patients might bring as many as 12500 out of the HGR group and 20000 people into the TGC group. This additional acquisition cost could be offset by relative savings on longer term cost reduction from, for example, lower rates of comorbidity. While the quoted NNT figures are not definitive, based on this modelling they provide a framework within which 


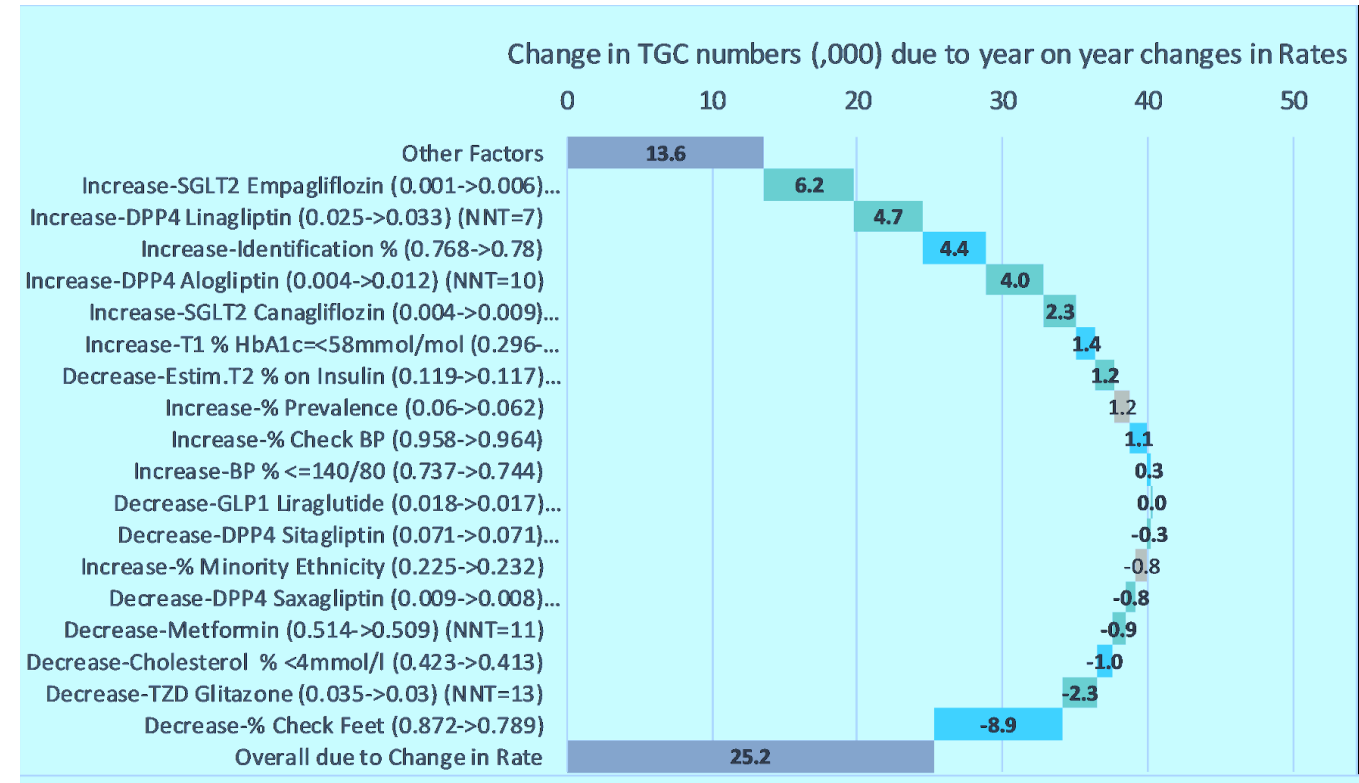

Change in HGR Numbers $(, 000)$ due to year on year changes in Rates $-20$

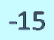

$-10$

$-5$

0

5

Other Factors

Increase-SGLT2 Empagliflozin (0.001->0.006) (NNT=6) Increase-SGLT2 Canagliflozin (0.004->0.009) (NNT=6)

Increase-DPP4 Linagliptin (0.025->0.033) (NNT $=12$ ) Increase-SGLT2 Dapagliflozin $(0.016->0.021)(\mathrm{NNT}=10)$ Increase-Identification $\%(0.768->0.78)$ Increase-GLP1 Dulaglutide (0->0.003) (NNT=6) Increase-DPP4 Alogliptin (0.004->0.012) (NNT=20) Increase-T1 \% HbA1c $=<58 \mathrm{mmol} / \mathrm{mol}(0.296->0.323)$

Decrease-DPP4 Sitagliptin (0.071->0.071) (NNT=12) Decrease-\% Check Urine Albumin (0.671->0.662) Increase-\% Check HbA1c $(0.952->0.953)$

Decrease-DPP4 Vildagliptin $(0.004>0.004)$ (NNT=8) Increase-\% in Most Deprived IMD (0.234->0.236) Increase-\% Check Creatine (0.948->0.951)

Decrease-Cholesterol $\%<4 \mathrm{mmol} / \mathrm{l}(0.423->0.413)$

Decrease-TZD Glitazone $(0.035->0.03)($ NNT $=17)$ Decrease-Age \% $>80(0.16->0.138)$ Decrease-\% Check Feet $(0.872->0.789)$ Overall Due to Change in Rate

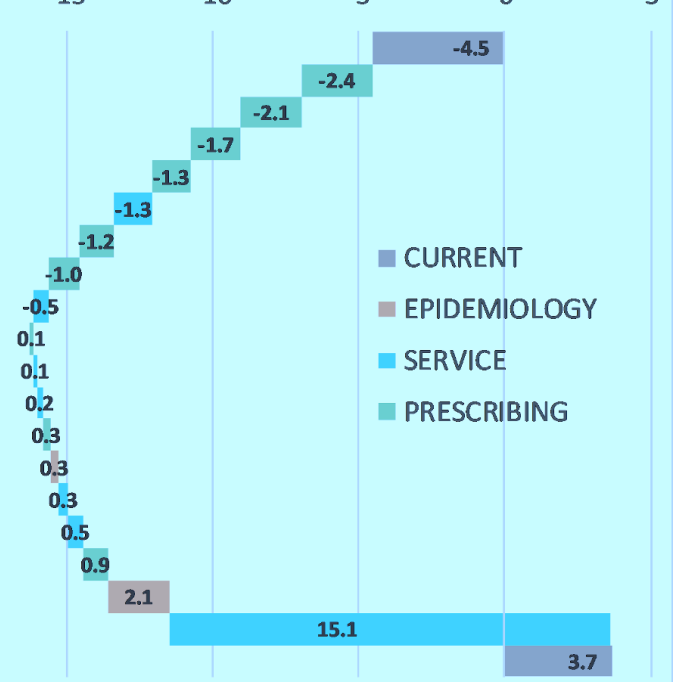

Figure 4 Reconciling actual change in numbers of TGC and HGR between 2015_16 and 2016_17 by applying the model regression coefficients $s$ to the annual change in value of the selected factors and then multiplying the result by the national T2DM population, along with Numbers Needed to Treat (NNT). BP, blood pressure; DPP-4, dipeptidyl peptidase-4 inhibitor; GLP-1, glucagon-like peptide-1 agonist; HGR, higher glycaemic risk; SGLT-2, sodium-glucose cotransporter-2 inhibitor; TGC, target glycaemic control

changes in clinical prescribing can be more accurately defined.

We accept that the methodology applied is relatively new and that the findings concerning the relative effect of different diabetes treatments need to be replicated in other T2DM populations. Nevertheless, the authors believe these results make an important contribution to the debate about potential impact of different prescribing and service activity approaches on glycaemic control.

\section{CONCLUSION}

The methodology described here gives the opportunity for GPP to evaluate their effectiveness to manage T2DM in the light of three key categories of 'medicines', 'service activities' and 'demographics'. The findings from this study emphasise the importance of practice level organisation of testing/recalland of care processes, and the importance of proactively reviewing and 'fine tuning' their prescribing behaviour more in line with higher performing practices, with the goal of improved outcomes for their T2DM patients.

GPP using more legacy therapies such as sulphonylurea/insulin attain poorer outcomes, while those applying holistic patient management and use of newer molecules are associated with improved outcomes. If all GPP adopted similar service activities and prescribing practices to those of the top decile for both TGC and HGR groups, outcomes could be substantially improved. 
Our findings demonstrate that the range of factors in the primary care management of T2DM, including monitoring and prescribing, can have significant positive and in some cases negative impact on patient outcomes, allowing for potential causal confounding factors. Understanding the specific relative contribution each factor makes to patient outcomes provides an important starting point for a more accurate prioritisation of clinical and funding activities, and therefore improved management of everyone with T2DM. Looking ahead, these findings can form the basis of an 'associated best practice' benchmark against which GPP could be evaluated and areas for improvement more easily identified.

\author{
Author affiliations \\ ${ }^{1}$ Department of Diabetes and Endocrinology, Salford Royal NHS Foundation Trust, \\ Salford, UK \\ ${ }^{2}$ The School of Medicine and Manchester Academic Health Sciences Centre, The \\ University of Manchester, Manchester, UK \\ ${ }^{3}$ Res Consortium, Andover, UK \\ ${ }^{4}$ Clinical Biochemistry, Walsall Healthcare NHS Trust, Walsall, UK \\ ${ }^{5}$ Clinical Biochemistry, University Hospitals of North Midlands, Stoke on Trent, \\ Staffordshire, UK \\ ${ }^{6}$ Warwick Medical School, University of Warwick, Coventry, UK
}

Contributors AH, MD, MS, MLi, MLu, AF and RG contributed to the conception, construction and writing of this paper. MS compiled the data set and undertook the data analysis.

Funding The authors have not declared a specific grant for this research from any funding agency in the public, commercial or not-for-profit sectors.

Competing interests None declared.

Patient consent for publication Not required.

Provenance and peer review Not commissioned; externally peer reviewed.

Data sharing statement All the data used for the analysis are available through the designated resources as outlined in the paper.

Open access This is an open access article distributed in accordance with the Creative Commons Attribution Non Commercial (CC BY-NC 4.0) license, which permits others to distribute, remix, adapt, build upon this work non-commercially, and license their derivative works on different terms, provided the original work is properly cited, appropriate credit is given, any changes made indicated, and the use is non-commercial. See: http://creativecommons.org/licenses/by-nc/4.0/.

\section{REFERENCES}

1. National Diabetes Audit (2017) http://content.digital.nhs.uk/diabetes inpatientaudit (last Accessed 17 Oct 2018)

2. NHS Digital. Prescribing for Diabetes in England - 2006/07-2016/17. 2017 https://digital.nhs.uk/data-and-information/publications/ statistical/prescribing-for-diabetes/prescribing-for-diabetes-england2006-07-to-2016-17 (last Accessed 17 Oct 2018).

3. Heald AH, Fryer AA, Anderson SG, et al. Sodium-glucose cotransporter-2 inhibitors, the latest residents on the block: Impact on glycaemic control at a general practice level in England. Diabetes Obes Metab 2018;20-1659-69.

4. NHS Digital. Quality and Outcomes Framework. $2018 \mathrm{https}: / /$ digital. nhs.uk/data-and-information/data-collections-and-data-sets/datacollections/quality-and-outcomes-framework-qof (last Accessed 20 May 2018).

5. NHS Digital. Primary Care Prescribing data base. 2017 https://digital. nhs.uk/prescribing (last Accessed 20 Oct 2018).

6. HERC. Yorkshire and the Number Public Health Observatory Data. 2018 https://www.herc.ox.ac.uk/./health_datasets/.data./yorkshireand-the-humber-public-health-observatory-indicator-search-tool (last Accessed 20 Oct 2018).

7. WHO. Defined Daily Dose (DDD): The assumed average maintenance dose per day for a drug used for its main indication in adults. 2018 http://www.who.int/medicines/regulation/medicines-safety/toolkit_ ddd/en/ (last accessed 20.10.18).

8. Heald AH, Livingston M, Malipatil N, et al. Improving type 2 diabetes mellitus glycaemic outcomes is possible without spending more on medication: Lessons from the UK National Diabetes Audit. Diabetes Obes Metab 2018;20:1-10.

9. Starks $\mathrm{H}$, Diehr P, Curtis JR, et al. The challenge of selection bias and confounding in palliative care research. J Palliat Med 2009;12:181-7.

10. Vasilakou D, Karagiannis T, Athanasiadou E, et al. Sodium-glucose cotransporter 2 inhibitors for type 2 diabetes: a systematic review and meta-analysis. Ann Intern Med 2013;159:262-74.

11. Wilding JP, Woo V, Rohwedder K, et al. Dapagliflozin in patients with type 2 diabetes receiving high doses of insulin: efficacy and safety over 2 years. Diabetes Obes Metab 2014;16:124-36.

12. Cha SA, Park YM, Yun JS, et al. A comparison of effects of DPP-4 inhibitor and SGLT2 inhibitor on lipid profile in patients with type 2 diabetes. Lipids Health Dis 2017;16:58.

13. Heald $\mathrm{AH}$, Livingston $\mathrm{M}$, Bien $\mathrm{Z}$, et al. The pattern of prescribing of glucose modulating agents for type 2 diabetes in general practices in England 2016/17. Int J Clin Pract 2018;72:e13080.

14. Heald $A H$, Livingston M, Fryer A, et al. Route to improving Type 1 diabetes mellitus glycaemic outcomes: real-world evidence taken from the National Diabetes Audit. Diabet Med 2018;35:63-71. 